\title{
Baseline requirements for detecting biosignatures with the HabEx and LUVOIR mission concepts
}

Ji Wang, Dimitri Mawet, Garreth Ruane, Jacques-Robert Delorme, Nikita Klimovich, et al.

Ji Wang, Dimitri Mawet, Garreth Ruane, Jacques-Robert Delorme, Nikita Klimovich, Renyu Hu, "Baseline requirements for detecting biosignatures with the HabEx and LUVOIR mission concepts," Proc. SPIE 10400, Techniques and Instrumentation for Detection of Exoplanets VIII, $104000 Z$ (1 September 2017); doi: 10.1117/12.2275222

SPIE Event: SPIE Optical Engineering + Applications, 2017, San Diego, California, United States 


\title{
Baseline Requirements For Detecting Biosignatures with the HabEx and LUVOIR Mission Concepts
}

\author{
Ji Wang $^{a}$, Dimitri Mawet ${ }^{a, b}$, Garreth Ruane $^{a}$, Jacques-Robert Delorme ${ }^{a}$, Nikita Klimovich ${ }^{a}$, \\ and Renyu $\mathrm{Hu}^{b, c}$ \\ ${ }^{a}$ Department of Astronomy, California Institute of Technology, Pasadena, CA 91125, USA; \\ ${ }^{b}$ Jet Propulsion Laboratory, California Institute of Technology, Pasadena, CA 91109, USA; \\ ${ }^{c}$ Division of Geological and Planetary Sciences, California Institute of Technology, Pasadena, \\ CA 91125, USA
}

\begin{abstract}
A milestone in understanding life in the universe is the detection of biosignature gases in the atmospheres of habitable exoplanets. Future mission concepts under study by the 2020 decadal survey, e.g., HabEx and LUVOIR, have the potential of achieving this goal. We investigate the baseline requirements for detecting four molecular species, $\mathrm{H}_{2} \mathrm{O}, \mathrm{O}_{2}, \mathrm{CH}_{4}$, and $\mathrm{CO}_{2}$. These molecules are highly relevant to habitability and life activity on Earth and other planets. Through numerical simulations, we find the minimum requirement for spectral resolution $(\mathrm{R})$ and starlight suppression level $(\mathrm{C})$ for a given exposure time. We consider scenarios in which different molecules are detected. For example, $\mathrm{R}=6400(400)$ and $\mathrm{C}=5 \times 10^{-10}\left(2 \times 10^{-9}\right)$ are required for HabEx (LUVOIR) to detect $\mathrm{O}_{2}$ and $\mathrm{H}_{2} \mathrm{O}$ for an exposure time of 400 hours for an Earth analog around a solar-type star at a distance of 5 pc. The full results are given in Table 2. The impact of exo-zodiacal contamination and thermal background is also discussed.
\end{abstract}

Keywords: HabEx, LUVOIR, Biosignature, Life, High Dispersion Coronagraphy

\section{INTRODUCTION}

Thousands of exoplanets have been discovered to date and many more will be detected by future missions. The focus of exoplanet studies are shifting towards understanding the statistical properties of exoplanets as a population and detailed characterization for nearby and scientifically-compelling individual systems. The primary goal of the latter is to study the chemical composition of exoplanet atmospheres and the implications for habitability and life activity.

Habitability requires a surface temperature that is suitable for life and the existence of liquid water $\left(\mathrm{H}_{2} \mathrm{O}\right)$ on the surface. Therefore, $\mathrm{H}_{2} \mathrm{O}$ is a primary molecular species to search for with future telescopes. In addition, biosignature gases, such as oxygen $\left(\mathrm{O}_{2}\right)$ and methane $\left(\mathrm{CH}_{4}\right)$, are highly indicative of life activity when coexisting out of thermodynamic equilibrium. ${ }^{1}$ Carbon dioxide $\left(\mathrm{CO}_{2}\right)$ is the most prominent greenhouse gas. A high concentration of $\mathrm{CO}_{2}$ may produce abiotic $\mathrm{O}_{2}$, so only low abundance of $\mathrm{CO}_{2}$ is suggestive of potential habitability. ${ }^{2}$ Future missions to search for and characterize habitable planets will have the ability to identify biosignature gases. However, there are several outstanding technical challenges:

- Aperture size of future space missions will be marginally adequate to identify certain biosignature gases. For example, as shown in this paper, it is extremely challenging to detect $\mathrm{CH}_{4}$.

- Despite much stronger biosignature signals in the near infrared, it is not likely that future large aperture space missions (4m-6.5m HabEx and 8m-16m LUVOIR) will have the capability to detect wavelengths larger than $1.8 \mu \mathrm{m}$. Otherwise, complicated cooling systems would be required to sufficiently reduce the thermal background, which may drastically increase the cost of the mission.

Further author information: e-mail: ji.wang@caltech.edu, telephone: +1-626-395-4981

Techniques and Instrumentation for Detection of Exoplanets VIII, edited by Stuart Shaklan, Proc. of SPIE Vol. 10400, 104000Z · C 2017 SPIE · CCC code: 0277-786X/17/\$18 · doi: 10.1117/12.2275222 


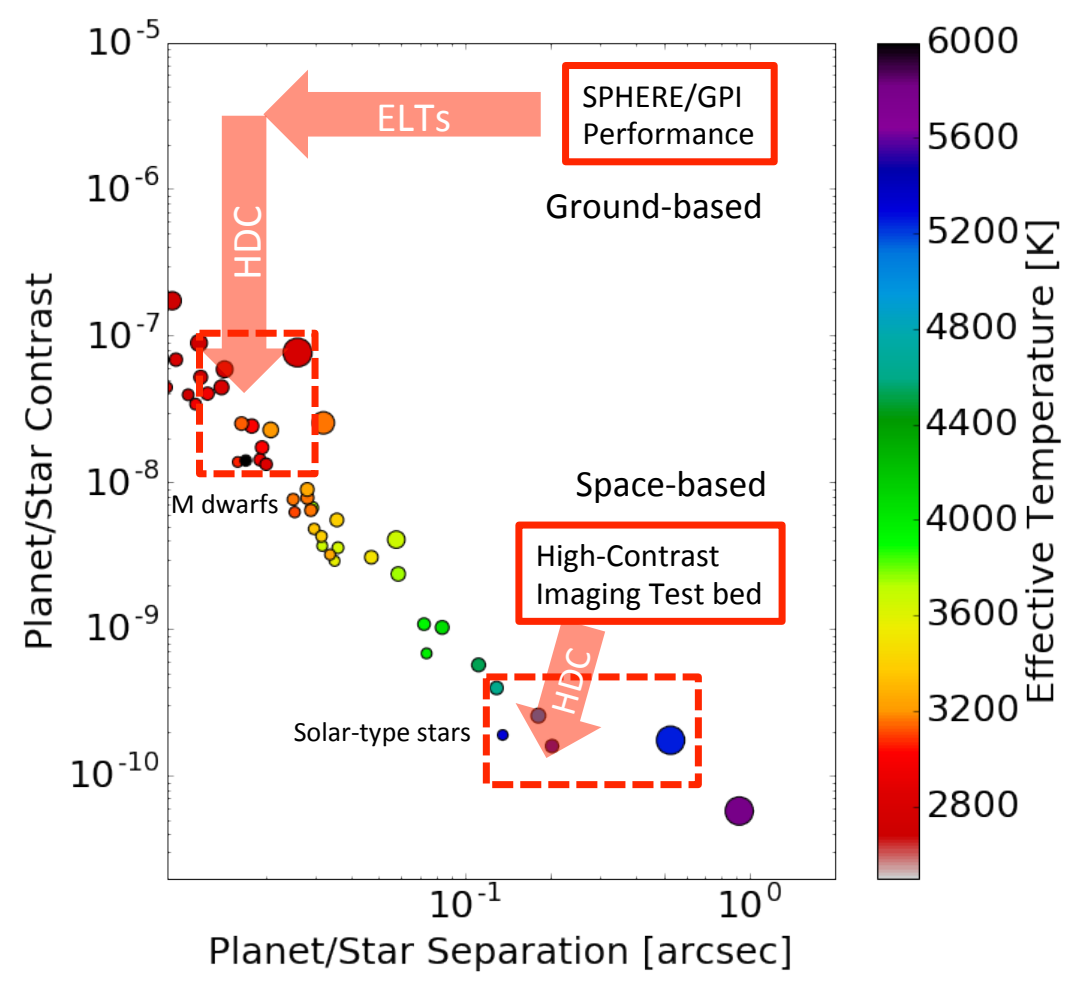

Figure 1. Planet/star contrast and angular separation for a hypothetical Earth-sized planet in the habitable zone around each star within 5 pc. Each data point is colored by host star effective temperature and the size is scaled with distance. Red solid-lined boxes mark state-of-the-art performance for ground-based direct imaging instruments and test beds for future space-based missions. Red dashed-line boxes indicate the notional objectives for future ground-based missions (i.e., planets around $\mathrm{M}$ dwarfs) and for future space missions (i.e., planets around solar-type stars)

- Identifying biosignature gases requires spectroscopic analysis of light from exoplanets. Most proposed dispersing elements for space missions to date have relatively low spectral resolution, e.g., $\mathrm{R} \sim 70$ for

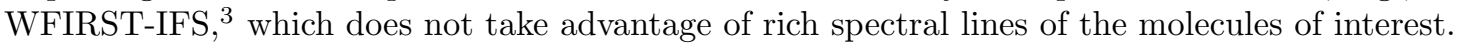

- Ground-based extremely large telescopes (ELTs) or Giant Segment Mirror Telescopes (GSMTs) will face severe challenges in breaking the current starlight suppression floor at $10^{-5}-10^{-6}$ level, which is set by adaptive optics system temporal bandwidth to correct for the Earth's atmospheric turbulence.

Fig. 1 summarizes the state-of-the-art performance of direct imaging instruments or testbeds (solid-lined boxes) and the requirements to detect a habitable planet (dashed-lined boxes). For both ground-based and space-based missions, there is a contrast gap between state-of-the-art performance and the planet/star contrast objective.

High dispersion coronagraphy (HDC) is a recent developed technique designed to bridge the contrast gap. ${ }^{4}$ HDC combines high contrast imaging (HCI), a single-mode fiber injection unit (FIU), ${ }^{5}$ and high resolution spectroscopy (HRS) to filter out stellar light and extract the planet's signal. Specifically, HCI suppresses stellar light and spatially separates the planet from its host star. The FIU filters out stellar noise at the planet location since the electric field of a stellar speckles does not couple to the fundamental mode of a single-mode fiber, whereas up to $\sim 80 \%$ of the planet light couples into the fiber. HRS further distinguishes planet signal from stellar signal by its unique spectral features such as absorption lines and radial velocity. Using this three-pronged starlight suppression, HDC can achieve the high sensitivity required to study terrestrial planets in the habitable zone. 
This paper focuses on the application of HDC on future space telescopes, such as HabEx and LUVOIR. The fundamental question we attempt to address here is whether these missions can detect biosignature gases $\left(\mathrm{O}_{2}\right.$ and $\left.\mathrm{CH}_{4}\right), \mathrm{H}_{2} \mathrm{O}$, and a false alarm indicator: $\mathrm{CO}_{2}$, and present mission requirements to achieve this goal. The four molecular species chosen have a relatively high concentration in the Earth's atmosphere and and are significant to habitability. Other biosignature gases, which have lower concentrations, are likely more difficult to detect. For example, only a few of the gases produced by life on Earth - $\mathrm{O}_{2}, \mathrm{O}_{3}, \mathrm{CH}_{4}$, and $\mathrm{N}_{2} \mathrm{O}$ - have been detected in earthshine and spacecraft observations of our own planet. ${ }^{6}$ Detecting these biosignature gases on another planet is expected to be far more challenging.

The paper is organized as follows. In $\S 1$, we briefly describe the four molecular species we investigate in this paper and the model we use to generate the spectra for our HDC simulations. In $\S 3$, we briefly describe our HDC simulation approach. More details can be found in previous work on HDC. ${ }^{4,7}$ Results are given in $\S 4$ followed by summary and conclusion in $\S 5$.

\section{MOLECULAR SPECIES OF INTEREST}

\subsection{Model Description}

The spectra of Earth-like exoplanets are generated by an atmospheric chemistry and radiative transfer model. ${ }^{8-11}$ We calculate the molecular abundance as a function of altitude, controlled by photochemical and disequilibrium chemistry processes. ${ }^{8}$ We include the effects of clouds in the resulting spectra by averaging two scenarios: a cloudfree scenario where we assume a clear atmosphere and a high-cloud scenario where we assume a reflective $\mathrm{H}_{2} \mathrm{O}$ cloud layer at 9-13 km. This procedure produces a continuum albedo of $\sim 0.3$ and provides a realistic estimate of the strength of spectral features. ${ }^{1}$ Eighth-order Gaussian integration is used to calculate the contribution of the whole planetary disk for both the reflected light and thermal emission. We include the opacities of $\mathrm{CO}_{2}, \mathrm{O}_{2}$, $\mathrm{H}_{2} \mathrm{O}$, and $\mathrm{CH}_{4}$ and calculate the planetary flux at a spectral resolution of $R=\lambda / \Delta \lambda=500,000$, which is high enough to resolve individual spectral lines of the aforementioned species over $\lambda=0.5-5 \mu \mathrm{m}$.

\subsection{Earth Spectrum By Molecule}

We consider four molecular species in an Earth's atmosphere, $\mathrm{CO}_{2}, \mathrm{O}_{2}, \mathrm{H}_{2} \mathrm{O}$, and $\mathrm{CH}_{4}$. Their spectra at different spectral resolutions are shown in Fig. 2. Observable line density and line depth decrease as spectral resolution decreases, making detecting certain molecules difficult at low spectral resolutions. Conversely, planet signal is dispersed to more pixels at high spectral resolutions. In such a case, molecule detectability is generally limited by detector noise. Since there are a number of zero- or low-noise detectors that potentially allow us to overcome the detector noise limited case, ${ }^{12}$ we assume noiseless detectors in the HDC simulations in this paper.

Fig. 2 also emphasizes the need of going beyond $1 \mu \mathrm{m}$ in order to detect biosignature gases. There are no strong lines of $\mathrm{CH}_{4}$ and $\mathrm{CO}_{2}$ below $1 \mu \mathrm{m}$. Detecting these two molecules is difficult at optical wavelengths. Even for molecules with strong lines below $1 \mu \mathrm{m}$, going above this wavelength would allow many more strong lines to be measured, thus increasing the detectability. We limit our HDC simulations below $1.8 \mu \mathrm{m}$, beyond which a cryogenic space mission would be required.

\section{SIMULATIONS OF HDC OBSERVATIONS}

\subsection{Methodology}

In this section, we briefly describe the procedure used to simulate HDC observations and data reduction. For more details in HDC simulations, please refer to two previous references. ${ }^{4,7}$

The planet and stellar spectra are convolved with a kernel that corresponds to a certain spectral resolution. The stellar signal is reduced by a factor we refer to as the starlight suppression level. The starlight suppression includes the suppression from the coronagraph, wavefront control, and additional nulling boost provided by the use of single-mode fibers. ${ }^{5}$

Poisson noise is added to account for photon noise. In addition, noise incurred in data reduction is included; e.g., errors associated with removing stellar light measured by additional fibers in speckle field. One particularly important noise source, speckle chromatic noise, is also taken into account. This noise arises from wavefront 

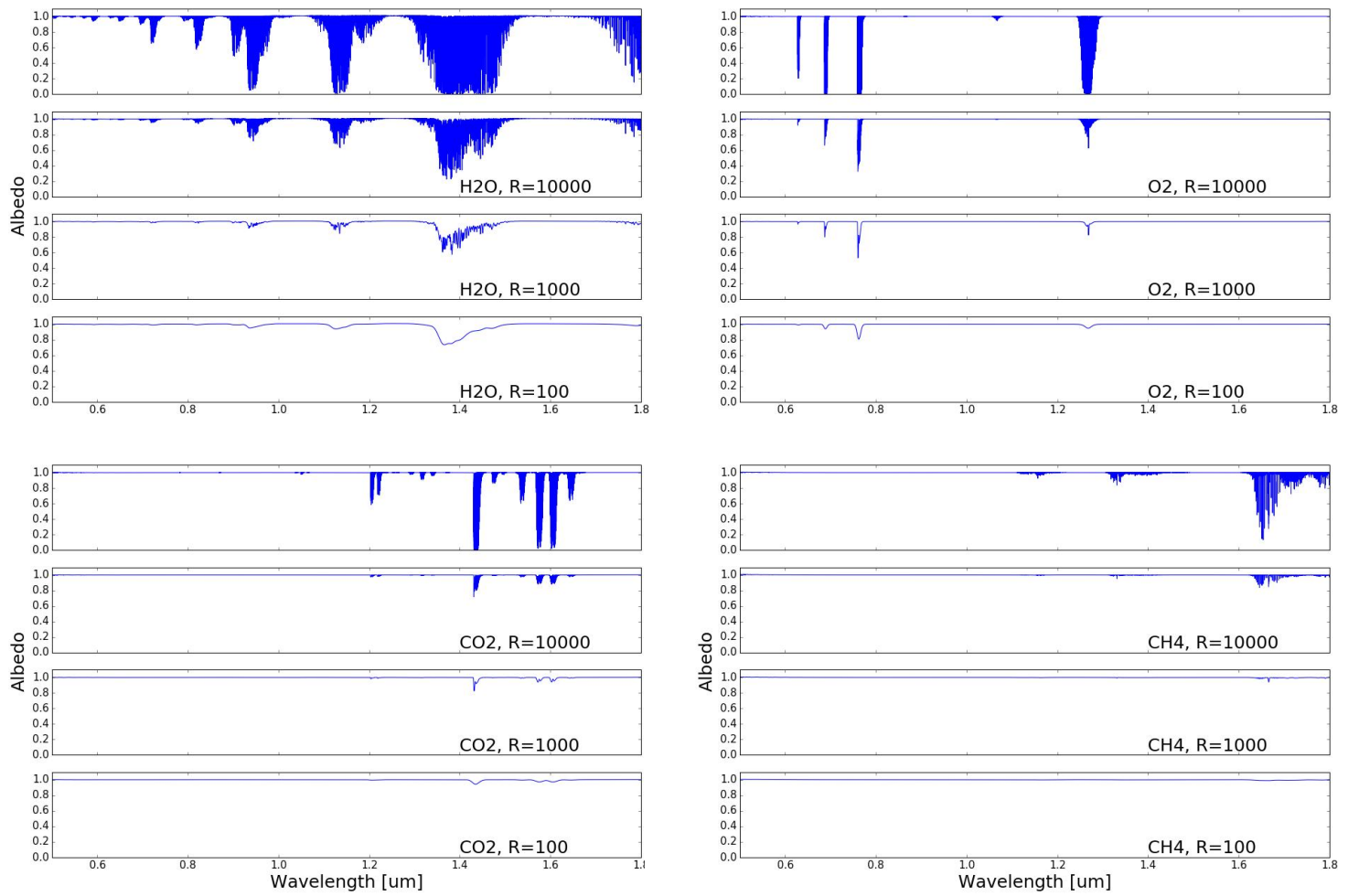

Figure 2. Normalized albedo spectra for $\mathrm{H}_{2} \mathrm{O}$ (top-left) $\mathrm{O}_{2}$ (top-right), $\mathrm{CO}_{2}$ (bottom-left), and $\mathrm{CH}_{4}$ (bottom-right). Each panel has 4 rows to show the influence of spectral resolution on spectral features. Top row is for R $>500,000$ followed by $\mathrm{R}$ of 10000,1000 , and 100, respectively.

control at high starlight suppression levels and prevents us from detecting biosignature gases at low spectral resolutions. ${ }^{4}$

The processed spectrum is then cross correlated with a template spectrum for the molecular species of interest and at the same spectral resolution as the observation. The resulting cross correlation function (CCF) is used for biosignature detection and to access the relative abundance of different molecules that are present in planet's atmosphere. The key parameters used in HDC simulations are given in Table 1.

Table 1. Inputs for HDC simulations for a Sun - Earth System

\begin{tabular}{|l|l|l|l|l|l|}
\hline \multicolumn{2}{|c|}{ Telescope/Instrument } & \multicolumn{2}{c|}{ Star } & \multicolumn{2}{c|}{ Planet } \\
\hline Telescope Aperture & $4 \mathrm{~m}$ or $12 \mathrm{~m}$ & $T_{\text {eff }}$ & $5800 \mathrm{~K}$ & Contrast & $6 \times 10^{-11}$ \\
\hline End-to-End Throughput & $10 \%$ & $\log (\mathrm{g})$ & 4.5 & Planet Radius & $1.0 \mathrm{R}_{\oplus}$ \\
\hline Spectral Resolution & varied & $V \sin i$ & $2.7 \mathrm{~km} / \mathrm{s}$ & $V \sin i$ & $0.5 \mathrm{~km} / \mathrm{s}$ \\
\hline Exposure Time & varied & Orbital Inclination & $50 \mathrm{deg}$ & Orbital Phase & 0.25 \\
\hline Wavelength & $0.5-1.8 \mu \mathrm{m}$ & Radial Velocity & $0.0 \mathrm{~km} / \mathrm{s}$ & Radial Velocity & $20.4 \mathrm{~km} / \mathrm{s}$ \\
\hline Detector Noise & 0 & Distance & $5 \mathrm{pc}$ & Semi-major Axis & $1 \mathrm{AU}$ \\
\hline
\end{tabular}

\subsection{Detection Definition}

Each HDC simulation results in a CCF. We repeat the HDC simulation 100 times for each combination of spectral resolution and starlight suppression level. We define detection of a certain molecular species with a set of criteria: 


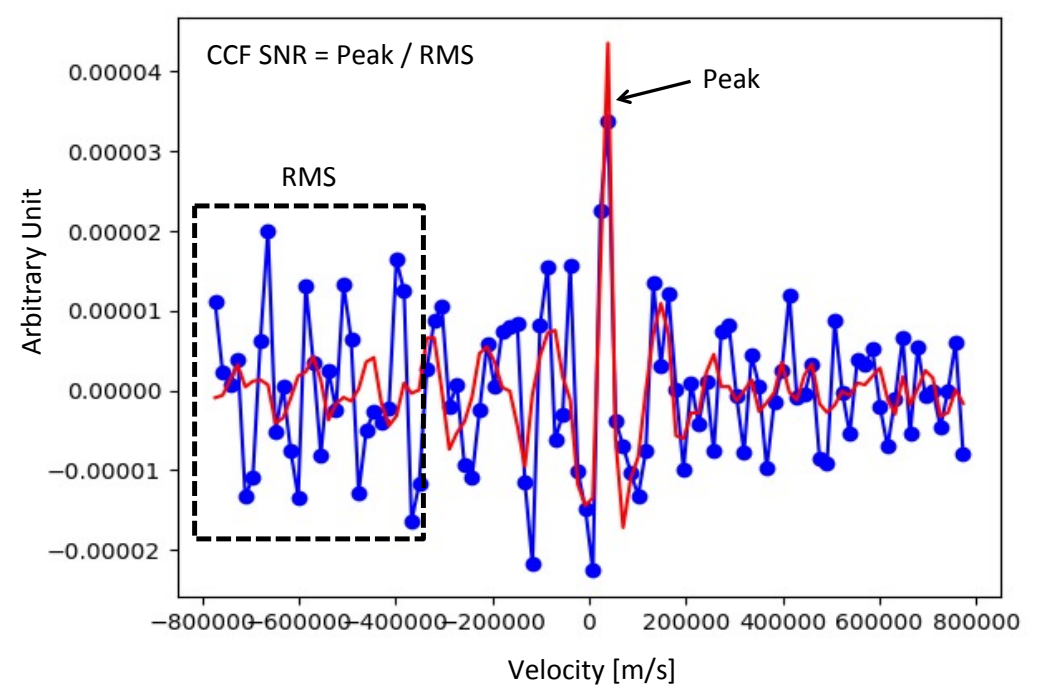

Figure 3. Example CCF in HDC simulation. Red CCF is for the noiseless case. Blue CCF with data points is for the case with noise added.

1. CCF SNR is higher than 3.0. CCF SNR is the ratio of the CCF value at planet radial velocity and root mean square (RMS) error calculated over large radial velocities where a peak is extremely unlikely (Fig. $3)$.

2. At least $68 \%$ of CCF SNRs of the 100 HDC simulations are higher than 3.0. This ensures that the majority of observations result in a higher than $3-\sigma$ detection.

3. The distribution of CCF SNR is significantly deviated from zero (Fig. 4). This limits the number of false negatives.

Here we use a different definition of detection from what was used in previous work. ${ }^{4}$ The previous definition concerns only the distribution of CCF SNR (criterion 2 and 3) but not the absolution CCF SNR (criterion 1). The new definition is therefore more conservative.

\section{RESULTS}

In this section, we summarize the requirements for detecting certain molecular species in terms of exposure time, spectral resolution, and starlight suppression level. Listed in order of decreasing difficulty, the molecules of interest are: $\mathrm{CH}_{4}, \mathrm{CO}_{2}, \mathrm{O}_{2}$, and $\mathrm{H}_{2} \mathrm{O}$. Therefore, other molecules will be detected if the minimum requirement is met for a more challenging molecule. We provide different scenarios for the HabEx and LUVOIR missions. Specifically, we discuss requirements for detection of (1) all four molecular species; (2) $\mathrm{CO}_{2}, \mathrm{O}_{2}$ and $\mathrm{H}_{2} \mathrm{O}$; and (3) $\mathrm{O}_{2}$ and $\mathrm{H}_{2} \mathrm{O}$ only. Each of these scenarios have different minimum requirements.

\subsection{Requirements for HabEx}

\subsubsection{Minimum Requirement for $\mathrm{CH}_{4}$ Detection}

Detection of $\mathrm{CH}_{4}$ requires a minimum spectral resolution of 6400 and starlight suppression level of $2 \times 10^{-11}$ if the maximum allowable exposure time is 4000 hours (Table 2).

Higher spectral resolution does not relax the starlight suppression requirement further. In principle, higher spectral resolution would result in a higher CCF peak. However, CCF RMS would increase too because more spectral features are resolved. In this case, CCF SNR is limited by the CCF's intrinsic structures. As a result, CCF SNR does not increase with increasing spectral resolution. One could remove the auto-correlation function 


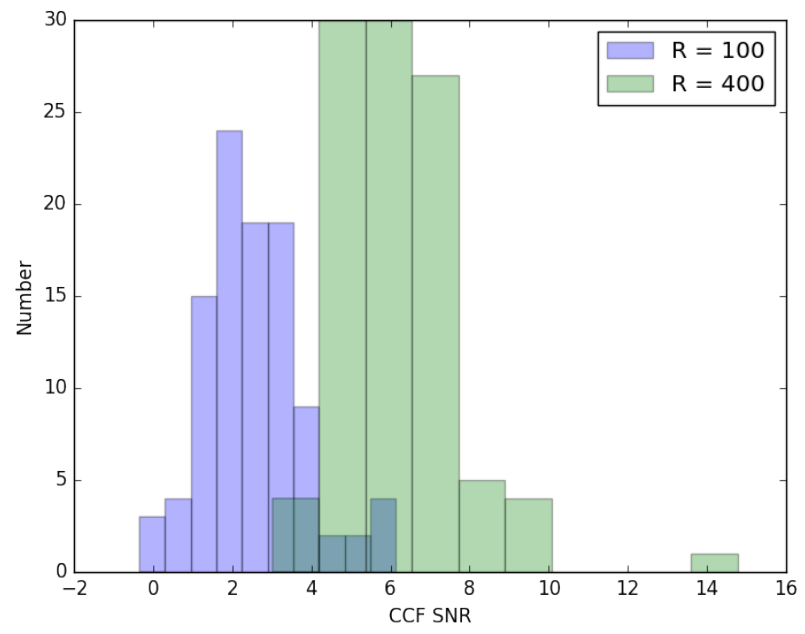

Figure 4. Distribution of CCF SNR at two spectral resolutions. Green histogram $(\mathrm{R}=400)$ is for the detection case because CCF SNR distribution is significantly deviated from zero whereas the blue histogram $(\mathrm{R}=100)$ is for the non-detection case.

Table 2. Minimum exposure time (in hours) for 3- $\sigma$ detection of a molecular species at a given combination of spectral resolution $(\mathrm{R})$ and starlight suppression level $(\mathrm{C})$. The results are for an Earth analog around a solar-type star at a distance of 5 pc. NA indicates that the molecular species is not considered in the search for biosignature gases.

\begin{tabular}{|l|l|l|l|l|l|}
\hline \multicolumn{7}{|c|}{$\mathrm{HabEx}$} \\
\hline $\mathrm{CH}_{4}$ & $\mathrm{CO}_{2}$ & $\mathrm{O}_{2}$ & $\mathrm{H}_{2} \mathrm{O}$ & $\mathrm{R}$ & $\mathrm{C}$ \\
\hline 4000.0 & 199.0 & 34.1 & 25.3 & 6400 & $2 \times 10^{-11}$ \\
\hline $\mathrm{NA}$ & 400.0 & 69.3 & 32.4 & 12800 & $5 \times 10^{-11}$ \\
\hline $\mathrm{NA}$ & $\mathrm{NA}$ & 400.0 & 108.9 & 6400 & $5 \times 10^{-10}$ \\
\hline \multicolumn{7}{|c|}{$\mathrm{LUVOIR}$} \\
\hline $\mathrm{CH}_{4}$ & $\mathrm{CO}_{2}$ & $\mathrm{O}_{2}$ & $\mathrm{H}_{2} \mathrm{O}$ & $\mathrm{R}$ & $\mathrm{C}$ \\
\hline 4000.0 & 307.2 & 52.6 & 28.7 & 6400 & $1 \times 10^{-9}$ \\
\hline 4000.0 & 178.2 & 10.2 & 7.3 & 800 & $5 \times 10^{-11}$ \\
\hline $\mathrm{NA}$ & 400.0 & 36.4 & 33.4 & 25600 & $1 \times 10^{-9}$ \\
\hline $\mathrm{NA}$ & 400.0 & 24.9 & 15.2 & 6400 & $5 \times 10^{-10}$ \\
\hline $\mathrm{NA}$ & 400.0 & 37.1 & 4.1 & 3200 & $1 \times 10^{-10}$ \\
\hline NA & 400.0 & 5.6 & 4.2 & 800 & $1 \times 10^{-11}$ \\
\hline NA & NA & 400.0 & 221.8 & 400 & $2 \times 10^{-9}$ \\
\hline
\end{tabular}

from the CCF to remove the CCF intrinsic structures. However, this operation is only effective when CCF SNR is high. This is not the case for what we consider here, i.e., 3- $\sigma$ detection.

At the spectral resolution of 6400 and starlight suppression level of $2 \times 10^{-11}$, the minimum exposure time for detection of $\mathrm{CO}_{2}, \mathrm{O}_{2}$, and $\mathrm{H}_{2} \mathrm{O}$ is 199.0, 34.1, and 25.3 hours, respectively (Table 2).

It is worth noting that the starlight suppression requirement level is $2 \times 10^{-11}$, which is lower than the planet/star contrast at $6 \times 10^{-11}$. This suggests that, while planet can be detected at $\sim 6 \times 10^{-11}$ a starlight suppression level, searching for $\mathrm{CH}_{4}$ would require a factor of three better starlight suppression and a much longer exposure time. 


\subsubsection{Minimum Requirement for $\mathrm{CO}_{2}$ Detection}

One may need to sacrifice the goal of $\mathrm{CH}_{4}$ detection if the minimum requirement is too challenging. In that case, biosignature search would focus on $\mathrm{CO}_{2}, \mathrm{O}_{2}$ and $\mathrm{H}_{2} \mathrm{O}$, in decreasing order of difficulty for detection. Detection of $\mathrm{CO}_{2}$ requires a minimum spectral resolution of 12800 and starlight suppression level of $5 \times 10^{-11}$ if the maximum allowable exposure time is 400 hours (Table 2). Detectable spectral features of $\mathrm{CO}_{2}$ are washed out as spectral resolution decreases (Fig. 2). Therefore, it is only detectable for spectral resolution higher than 12800.

At the spectral resolution of 12800 and starlight suppression level of $5 \times 10^{-11}$, the minimum exposure time for detection of $\mathrm{O}_{2}$ and $\mathrm{H}_{2} \mathrm{O}$ is 69.3 and 32.4 hours (Table 2). In comparison to the $\mathrm{HabEx}_{\mathrm{CH}_{4}}$ case, the minimum exposure time increases because of increased amount of stellar contamination $\left(5 \times 10^{-11}\right.$ vs. $\left.2 \times 10^{-11}\right)$.

\subsubsection{Minimum Requirement for $\mathrm{O}_{2}$ Detection}

If a biosignature search is confined to only $\mathrm{O}_{2}$ and $\mathrm{H}_{2} \mathrm{O}$, detection of $\mathrm{O}_{2}$ requires a minimum spectral resolution of 6400 and starlight suppression level of $5 \times 10^{-10}$ if the maximum allowable exposure time is 400 hours (Table 2). The minimum exposure time for detection of $\mathrm{H}_{2} \mathrm{O}$ is 108.9 hours. This case has the lowest requirement for spectral resolution and starlight suppression at the cost of missing $\mathrm{CH}_{4}$ and $\mathrm{CO}_{2}$, which are two important molecular species for determining habitability.

\subsection{Requirements for LUVOIR}

\subsubsection{Minimum Requirement for $\mathrm{CH}_{4}$ Detection}

We present two sets of $\mathrm{R}-\mathrm{C}$ (resolution-contrast) requirements for detection of $\mathrm{CH}_{4}$ (Table 2). Given a maximum allowable exposure time of 4000 hours, spectral resolution of 6400 , a starlight suppression level of $1 \times 10^{-9}$ is required. At a spectral resolution of 800, a starlight suppression level of $5 \times 10^{-11}$ is required for $\mathrm{CH}_{4}$ detection.

In comparison with the $\mathrm{HabEx} \mathrm{CH}_{4}$ case, starlight suppression requirement is relaxed to $1 \times 10^{-9}$ because of a larger aperture (3 times) and thus a higher planet signal (9 times). While starlight suppression requirement is less stringent for the LUVOIR $\mathrm{CH}_{4}$ case $\left(1 \times 10^{-9}\right)$ than the HabEx $\mathrm{CH}_{4}$ case $\left(2 \times 10^{-11}\right)$, we note that HabEx can potentially achieve higher level of starlight suppression level than LUVOIR because the HabEx telescope will be optimized for coronagraphic observations.

There is another notable difference from the HabEx $\mathrm{CH}_{4}$ case. $\mathrm{CH}_{4}$ can be detected at $\mathrm{R}=800$, a much lower spectral resolution than $R=6400$. However, the spectral resolution and starlight suppression may be traded in the instrument design.

At the spectral resolution of 6400 and starlight suppression level of $1 \times 10^{-9}$, the minimum exposure times for detection of $\mathrm{CO}_{2}, \mathrm{O}_{2}$, and $\mathrm{H}_{2} \mathrm{O}$ are respectively 307.2, 52.6, and 28.7 hours. For another set of minimum requirements, $\mathrm{R}=800$ and $\mathrm{C}=5 \times 10^{-11}$, the minimum exposure time for detection of $\mathrm{CO}_{2}, \mathrm{O}_{2}$, and $\mathrm{H}_{2} \mathrm{O}$ is $178.2,10.2$, and 7.3 hours (Table 2). The minimum exposure time is further reduced with improved starlight suppression.

\subsubsection{Minimum Requirement for $\mathrm{CO}_{2}$ Detection}

The trade between spectral resolution and starlight suppression for $\mathrm{CO}_{2}$ detection is more complicated than the LUVOIR $\mathrm{CH}_{4}$ case. There are four R-C combinations that set the minimum requirements for detection of $\mathrm{CO}_{2}$ (Table 2). The minimum requirements follow the general trend of increasing spectral resolution with relaxed requirement for starlight suppression. As spectral resolution increases from 800 to 25600 , the requirement for starlight suppression is relaxed from $1 \times 10^{-11}$ to $1 \times 10^{-9}$.

\subsubsection{Minimum Requirement for $\mathrm{O}_{2}$ Detection}

If the biosignature search is only focused on $\mathrm{O}_{2}$ and $\mathrm{H}_{2} \mathrm{O}$, detection of $\mathrm{O}_{2}$ requires a minimum spectral resolution of 400 and starlight suppression level of $2 \times 10^{-9}$ if the maximum allowable exposure time is 400 hours (Table 2). The minimum exposure time for detection of $\mathrm{H}_{2} \mathrm{O}$ is 221.8 hours. 


\section{SUMMARY AND CONCLUSION}

We study the detectability of four molecular species as biosignature or habitability indicators: $\mathrm{CH}_{4}, \mathrm{CO}_{2}, \mathrm{O}_{2}$ and $\mathrm{H}_{2} \mathrm{O}$. We conduct HDC simulations to set the minimum requirement for detection in terms of exposure time, spectral resolution and starlight suppression level. Major findings are summarized in Table 2. The result provides a baseline for mission design in order to search for biosignature gases and study the habitability of exoplanets.

\subsection{Challenge in $\mathrm{CH}_{4}$ Detection}

Detection of $\mathrm{CH}_{4}$, an important biosignature gas, is extremely difficult. Given an exposure time of 4000 hours, the minimum starlight suppression level is $2 \times 10^{-11}$, which is extremely challenging to achieve even for a coronagraphy-friendly HabEx mission. The starlight suppression requirement is relaxed to $1 \times 10^{-9}$ for the LUVOIR $\mathrm{CH}_{4}$ case mainly due to a much larger aperture. However, given that HabEx is optimized for exoplanet detection whereas LUVOIR is more general purposed, it is unclear which case is more challenging to achieve: $1 \times 10^{-9}$ for LUVOIR or $2 \times 10^{-11}$ for HabEx. For both cases, an investment of 4000 hours exposure time would be expensive and may require further justification to ensure scientific return.

\subsection{Exo-Zodiacal Flux}

We consider in our HDC simulation only starlight suppression levels that expected to be achieved by a coronagraphic system. Starlight is reduced based on the suppression factor given in Table 2. However, the flux from exo-zodiacal dust may set a floor for the achievable starlight suppression level. This floor is estimated at a few times the planet/star contrast for a 4-m aperture, i.e., $\sim 10^{-10} .{ }^{13,14}$ If this is the case, then HabEx would only be able to detect $\mathrm{O}_{2}$ and $\mathrm{H}_{2} \mathrm{O}$. LUVOIR designs are less prone to exo-zodiacal flux because of a smaller point spread function and thus smaller contamination area. This is a result of the larger aperture of LUVOIR.

\subsection{Background From A Warm Telescope}

Another noise source that is not accounted for in our HDC simulation is the thermal background emission from the telescope and instrument. This is not a typical concern at optical wavelengths. For example, thermal background of a warm primary mirror $(270 \mathrm{~K}$, emissivity $=0.02)$ is comparable to the signal of a Earth analog at $5 \mathrm{pc}$ at $\sim 0.8 \mu \mathrm{m}$. However, thermal background quickly becomes a severe issue at longer wavelengths. For a cutoff wavelength at $1.8 \mu \mathrm{m}$, the instrument needs to be cooler than $130 \mathrm{~K}$ in order for the thermal background to be below the planet signal. Mission designs exist that cool the system to $130 \mathrm{~K}$ without active cryogenic cooling. For example, Euclid uses a passive cooling radiator system to cool the system to $130 \mathrm{~K} .{ }^{15}$ The telescope and instrument need to remain $¡ 100 \mathrm{~K}$ if the cutoff wavelength extends to $K$ band.

\section{ACKNOWLEDGMENTS}

We would like to thank Rowan Swain for proofreading the manuscript.

\section{REFERENCES}

[1] Des Marais, D. J., Harwit, M. O., Jucks, K. W., Kasting, J. F., Lin, D. N. C., Lunine, J. I., Schneider, J., Seager, S., Traub, W. A., and Woolf, N. J., "Remote Sensing of Planetary Properties and Biosignatures on Extrasolar Terrestrial Planets," Astrobiology 2, 153-181 (June 2002).

[2] Domagal-Goldman, S. D., Segura, A., Claire, M. W., Robinson, T. D., and Meadows, V. S., "Abiotic Ozone and Oxygen in Atmospheres Similar to Prebiotic Earth," ApJ 792, 90 (Sept. 2014).

[3] McElwain, M. W., Mandell, A. M., Gong, Q., Llop-Sayson, J., Brandt, T., Chambers, V. J., Grammer, B., Greeley, B., Hilton, G., Perrin, M. D., Stapelfeldt, K. R., Demers, R., Tang, H., and Cady, E., "PISCES: an integral field spectrograph technology demonstration for the WFIRST coronagraph," in [Space Telescopes and Instrumentation 2016: Optical, Infrared, and Millimeter Wave], SPIE 9904, 99041A (July 2016).

[4] Wang, J., Mawet, D., Ruane, G., Hu, R., and Benneke, B., "Observing Exoplanets with High Dispersion Coronagraphy. I. The Scientific Potential of Current and Next-generation Large Ground and Space Telescopes," AJ 153, 183 (Apr. 2017). 
[5] Mawet, D., Ruane, G., Xuan, W., Echeverri, D., Klimovich, N., Randolph, M., Fucik, J., Wallace, J. K., Wang, J., Vasisht, G., Dekany, R., Mennesson, B., Choquet, E., Delorme, J.-R., and Serabyn, E., "Observing Exoplanets with High-dispersion Coronagraphy. II. Demonstration of an Active Single-mode Fiber Injection Unit," ApJ 838(2), 92 (2017).

[6] Seager, S., Bains, W., and Petkowski, J. J., "Toward a List of Molecules as Potential Biosignature Gases for the Search for Life on Exoplanets and Applications to Terrestrial Biochemistry," Astrobiology 16, 465-485 (June 2016).

[7] Wang, J., Mawet, D., Hu, R., and Benneke, B., "High-contrast imaging and high-resolution spectroscopy observation of exoplanets," in [Modeling, Systems Engineering, and Project Management for Astronomy VI], SPIE 9911, 99112T (Aug. 2016).

[8] Hu, R., Ehlmann, B. L., and Seager, S., "Theoretical Spectra of Terrestrial Exoplanet Surfaces," ApJ 752, 7 (June 2012).

[9] Hu, R., Seager, S., and Bains, W., "Photochemistry in Terrestrial Exoplanet Atmospheres. I. Photochemistry Model and Benchmark Cases," ApJ 761, 166 (Dec. 2012).

[10] Hu, R., Seager, S., and Bains, W., "Photochemistry in Terrestrial Exoplanet Atmospheres. II. $\mathrm{H}_{2} \mathrm{~S}$ and $\mathrm{SO}_{2}$ Photochemistry in Anoxic Atmospheres," ApJ 769, 6 (May 2013).

[11] Hu, R. and Seager, S., "Photochemistry in Terrestrial Exoplanet Atmospheres. III. Photochemistry and Thermochemistry in Thick Atmospheres on Super Earths and Mini Neptunes," ApJ 784, 63 (Mar. 2014).

[12] Rauscher, B. J., Canavan, E. R., Moseley, S. H., Sadleir, J. E., and Stevenson, T., "Detectors and cooling technology for direct spectroscopic biosignature characterization," Journal of Astronomical Telescopes, Instruments, and Systems 2, 041212 (Oct. 2016).

[13] Roberge, A., Chen, C. H., Millan-Gabet, R., Weinberger, A. J., Hinz, P. M., Stapelfeldt, K. R., Absil, O., Kuchner, M. J., and Bryden, G., "The Exozodiacal Dust Problem for Direct Observations of Exo-Earths," PASP 124, 799 (Aug. 2012).

[14] Stark, C. C., Roberge, A., Mandell, A., and Robinson, T. D., "Maximizing the ExoEarth Candidate Yield from a Future Direct Imaging Mission," ApJ 795, 122 (Nov. 2014).

[15] Racca, G. D., Laureijs, R., Stagnaro, L., Salvignol, J.-C., Lorenzo Alvarez, J., Saavedra Criado, G., Gaspar Venancio, L., Short, A., Strada, P., Bönke, T., Colombo, C., Calvi, A., Maiorano, E., Piersanti, O., Prezelus, S., Rosato, P., Pinel, J., Rozemeijer, H., Lesna, V., Musi, P., Sias, M., Anselmi, A., Cazaubiel, V., Vaillon, L., Mellier, Y., Amiaux, J., Berthé, M., Sauvage, M., Azzollini, R., Cropper, M., Pottinger, S., Jahnke, K., Ealet, A., Maciaszek, T., Pasian, F., Zacchei, A., Scaramella, R., Hoar, J., Kohley, R., Vavrek, R., Rudolph, A., and Schmidt, M., "The Euclid mission design," in [Space Telescopes and Instrumentation 2016: Optical, Infrared, and Millimeter Wave], SPIE 9904, $99040 \mathrm{O}$ (July 2016). 